\title{
Identification of Angelica acutiloba and Related Species by Analysis of Inter- and Intra-Specific Sequence Variations in Chloroplast and Nuclear DNA Sequences
}

\author{
Kiyoshi Matsubara*, Satoshi Shindo, Hitoshi Watanabe, Fumio Ikegami \\ Center for Environment, Health and Field Sciences, Chiba University, Kashiwa, Japan. \\ Email: *k-matsubara@faculty.chiba-u.jp
}

Received July 31 ${ }^{\text {st }}$, 2012; revised August $26^{\text {th }}, 2012$; accepted September $5^{\text {th }}, 2012$

\begin{abstract}
Japanese Angelica Root prepared from Angelica acutiloba var. acutiloba and A. acutiloba var. sugiyamae, known in Japan as "Toki" and "Hokkai Toki", is an important crude drug used in Kampo medicine (traditional Japanese medicine). However, since these Angelica varieties have recently outcrossed with each other, it is unclear whether Japanese Angelica Root sold for use in Kampo medicine is a pure variety. Here, we describe DNA sequence polymorphisms that can be used to distinguish between A. acutiloba var. acutiloba and A. acutiloba var. sugiyamae. In our analyses, differences in the trnK region of chloroplast DNA distinguished among some A. acutiloba varieties and related species, but not between A. acutiloba var. acutiloba and A. acutiloba var. iwatensis. One geographical strain of A. acutiloba var. acutiloba and A. acutiloba var. sugiyamae showed identical sequences in three regions of chloroplast DNA, but differences in the internal transcribed spacer region of nuclear ribosomal DNA. One strain of A. acutiloba var. iwatensis and A. acutiloba var. sugiyamae had identical sequences in all of the chloroplast and nuclear ribosomal DNA regions examined. These findings show that A. acutiloba var. acutiloba has hybridized with A. acutiloba var. sugiyamae and that the "Hokkai Toki" variety resulted from outcrossing with A. acutiloba var. iwatensis. Molecular authentication based on analyses of chloroplast and nuclear ribosomal DNA sequences of A. acutiloba and related species is an efficient method to authenticate Japanese Angelica Root at the variety level. Therefore, these analyses can determine whether a product is derived from A. acutiloba var. acutiloba or A. acutiloba var. sugiyamae.
\end{abstract}

Keywords: Angelica acutiloba; Chloroplast DNA; ITS; Japanese Angelica Root; Kampo Medicine; Sequence Variation

\section{Introduction}

The Japanese indigenous species of Angelica acutiloba Kitagawa var. acutiloba Kitagawa (Toki) or A. acutiloba Kitagawa var. sugiyamae Hikino (Hokkai Toki) are listed in the Japanese Pharmacopoeia, $16^{\text {th }}$ Edition [1], and are precious crude drugs in Kampo medicine (traditional Japanese medicine). The A. acutiloba cultivar (also called "Yamato Toki" or "Ohbuka Toki”), is of higher quality than the "Hokkai Toki" cultivar, and was originnally grown in Nara prefecture, Japan. The high quality of this cultivar is described in historical Japanese literature $[2,3]$. To this day, its quality is still regarded as being higher than that of other cultivars, and this is reflected in the market price of the root. Since the 1950s, the "Hokkai Toki" variety of A. acutiloba var. sugiyamae has been cultivated in various regions of Japan, including Nara prefecture [4]. Angelica species are thought to be

\footnotetext{
*Corresponding author.
}

outcrossed [5]. There are wild-growing species that that are closely related to A. acutiloba, including A. acutiloba Kitagawa var. iwatensis Hikino (Miyama Toki), which is genetically very close to "Yamato Toki", and A. stenoloba ("Hosoba Toki” or "Tokachi Toki”) [6]. These species readily outcross with A. acutiloba var. acutiloba and A. acutiloba var. sugiyamae [7].

Previous molecular studies on A. acutiloba did not show any nucleotide polymorphisms in the intergenic spacer region of 5S rDNA [8], while A. acutiloba var. iwatensis collected from different geographical locations showed genetic polymorphisms in a random amplified polymorphism (RAPD) analysis [9]. There were small differences in RAPD patterns between A. acutiloba var. acutiloba and A. acutiloba var. sugiyamae [10], and there were differences among individual plants of these varieties, indicating genetic diversity among A. acutiloba varieties. The three varieties of $A$. acutiloba var. acutiloba, A. acutiloba var. sugiyamae, and A. acutiloba var. iwat- 
ensis had two-nucleotide differences in the spacer region between the $a t p \mathrm{~F}$ and atpA genes in the plastid genome [11]. Although there are genomic DNA polymorphisms among Angelica varieties, these varieties are genetically heterogeneous because of the high rate of cross-pollination [12].

The root of A. acutiloba var. iwatensis is not used as a crude drug for Kampo medicine in Japan [1], although the morphological features are very similar among related varieties of $A$. acutiloba, making them difficult to distinguish from one another. Therefore, a simple method to discriminate among these varieties is required. A. acutiloba var. acutiloba is also cultivated in China [13]. The Chinese product prepared from the root of A. acutiloba var. acutiloba is called "Nisshiki Toki" and resembles the Japanese style of "Yamato Toki". This product has been imported into Japan. It is now difficult to find the original varieties of A. acutiloba var. acutiloba and A. acutiloba var. sugiyamae. Therefore, it is important to be able to distinguish among species and to determine the geographic origin of Angelica varieties. A reliable identification method will be useful to maintain pure varieties with superior traits for use in Kampo medicine.

In this study, we investigated various DNA sequences to provide useful information for authentication of $A$. acutiloba varieties and related species. The DNA sequences were the atpF-atpA, rpl16-rpl14, and trnK regions of chloroplast DNA (cpDNA), and the internal transcribed spacer (ITS) of nuclear ribosomal DNA, consisting of five regions; 18S, ITS1, 5.8S, ITS2, and 26S.

\section{Materials and Methods}

\subsection{Plant Materials}

As shown in Table 1, we collected nine strains of Angelica acutiloba var. acutiloba, three of A. acutiloba var. sugiyamae, and two of A. acutiloba var. iwatensis from cultivars of different location or native populations. We also collected the cultivated strains of related species $A$. stenoloba ("Hosoba Toki”), A. stenoloba f. lanceolata (“Tokachi Toki”), and A. shikokiana ("Inu Toki”). Each collected plant was identified by morphological characters. Three individuals of each strain/variety were cultivated in a greenhouse before use in experiments.

Table 1. Plant materials used in this study and summary of haplotype data for Angelica acutiloba and related species.

\begin{tabular}{|c|c|c|c|c|c|c|c|c|}
\hline \multirow{2}{*}{$\begin{array}{l}\text { Sample } \\
\text { code }\end{array}$} & \multirow{2}{*}{ Species } & \multirow{2}{*}{$\begin{array}{l}\text { Japanese } \\
\text { name }\end{array}$} & \multirow{2}{*}{ Locality } & \multirow{2}{*}{ Origin } & \multicolumn{4}{|c|}{ DDBJ/GenBank/EBI Data Bank accession number } \\
\hline & & & & & atpF-atpA & rpl16-rpl14 & $\operatorname{trnK}$ & ITS \\
\hline TG- $1^{\mathrm{a}}$ & A. acutiloba var. acutiloba & Toki & Gumma & Cultivar & AB697525 & AB697547 & AB697569 & AB697591 \\
\hline TG-2 & A. acutiloba var. acutiloba & Toki & Gumma & Cultivar & AB697526 & AB697548 & AB697570 & AB697592 \\
\hline TK & A. acutiloba var. acutiloba & Toki & Kyoto & Cultivar & AB697527 & AB697549 & AB697571 & AB697593 \\
\hline TY & A. acutiloba var. acutiloba & Toki & Yamagata & Cultivar & AB697528 & AB697550 & AB697572 & AB697594 \\
\hline $\mathrm{TH}$ & A. acutiloba var. acutiloba & Toki & Hokkaido & Cultivar & AB697529 & AB697551 & AB697573 & AB697595 \\
\hline TC & A. acutiloba var. acutiloba & Toki & China & Cultivar & AB697530 & AB697552 & AB697574 & AB697596 \\
\hline YH & A. acutiloba var. acutiloba & Toki & Hokkaido & Cultivar & AB697531 & AB697553 & AB697575 & AB697597 \\
\hline YM & A. acutiloba var. acutiloba & Toki & Miyagi & Cultivar & AB697532 & AB697554 & AB697576 & AB697598 \\
\hline OT-1 & A. acutiloba var. acutiloba & Toki & Toyama & Cultivar & AB697533 & AB697555 & AB697577 & AB697599 \\
\hline OT-2 & A. acutiloba var. acutiloba & Toki & Toyama & Cultivar & AB697534 & AB697556 & AB697578 & AB697600 \\
\hline OC-1 & A. acutiloba var. acutiloba & Toki & Chiba & Cultivar & AB697535 & AB697557 & AB697579 & AB697601 \\
\hline OC-2 & A. acutiloba var. acutiloba & Toki & Chiba & Cultivar & AB697536 & AB697558 & AB697580 & AB697602 \\
\hline HA & A. acutiloba var. sugiyamae & Hokkai Toki & Hokkaido & Cultivar & AB697537 & AB697559 & AB697581 & AB697603 \\
\hline HB & A. acutiloba var. sugiyamae & Hokkai Toki & Hokkaido & Cultivar & AB697538 & AB697560 & AB697582 & AB697604 \\
\hline $\mathrm{HC}$ & A. acutiloba var. sugiyamae & Hokkai Toki & Chiba & Cultivar & AB697539 & AB697561 & AB697583 & AB697605 \\
\hline MM & A. acutiloba var. iwatensis & Miyama Toki & Miyagi & Cultivar & AB697540 & AB697562 & AB697584 & AB697606 \\
\hline MY & A. acutiloba var. iwatensis & Miyama Toki & Yamagata & Native population & AB697541 & AB697563 & AB697585 & AB697607 \\
\hline $\mathrm{LH}$ & A. stenoloba & Hosoba Toki & Saitama & Cultivar & AB697542 & AB697564 & AB697586 & AB697608 \\
\hline $\mathrm{LT}$ & A. stenoloba f. lanceolata & Tokachi Toki & Saitama & Cultivar & AB697543 & AB697565 & AB697587 & AB697609 \\
\hline IM-1 & A. shikokiana & Inu Toki & Miyazaki & Cultivar & AB697544 & AB697566 & AB697588 & AB697610 \\
\hline IM-2 & A. shikokiana & Inu Toki & Miyazaki & Cultivar & AB697545 & AB697567 & AB697589 & AB697611 \\
\hline BB & Peucedanum japonicum & Botanbofu & Chiba & Native population & AB697546 & AB697568 & AB697590 & AB697612 \\
\hline
\end{tabular}

a -1 , and -2 are the same strain but different individuals. 


\subsection{Total DNA Extraction and Amplifications}

We extracted total DNA from approximately $200 \mathrm{mg}$ young leaf tissue using the modified CTAB method [14]. The extracted DNA was used as the template for polymerase chain reaction (PCR) amplifications. The atpFatpA, rpl16-rpl14, and trnK regions, and the entire ITS region containing intergenic spacer regions were amplified with the following primers: atpF1 (TTACGAGGAGCTCTAGAACTCTGAATAGTTGTT TG) and atpR1 (GCCATTACTTCATCAAGACCGTG AATACGAGCAATGCC) for the atpF-atpA region desinged by Hosokawa et al. [11]; PSIDF1 (AAAGATCTAGATTTCGTAAACAACATAGAGGAAGAA) and PSIDR1 (ATCTGCAGCATTTAAAAGGGTCTGAGGT TGAATCAT) for the rpl16-rpl14 region desinged by Ohta et al. [15]; trnKF1 (TGGGTTGCTAACTCAATG G) and trnKR1 (AACTAGTCGGATGGAGTAG) for the trnK region desinged by Zhu et al. [16]; and ITSF1 (TCCACTGAACCTTATCATTTAG) and ITSR1 (CCA TGCTTAAACTCAGCGGGT) for the ITS region desinged in this study. Each amplification was carried in a reaction mixture containing 50 ng total DNA, $1 \times$ ExTaq buffer, $0.2 \mathrm{mM}$ each deoxynucleotide triphosphate, $1 \mathrm{mM}$ each primer, and 2.5 U ExTaq (TaKaRa, Japan). The amplifycation conditions were as follows: initial denaturation for $5 \mathrm{~min}$ at $95^{\circ} \mathrm{C}$, followed by 30 cycles of $95^{\circ} \mathrm{C}$ for $45 \mathrm{~s}$, $60^{\circ} \mathrm{C}$ for $1 \mathrm{~min}$, and $72^{\circ} \mathrm{C}$ for $2 \mathrm{~min}$, and a final extension at $72^{\circ} \mathrm{C}$ for $5 \mathrm{~min}$. The PCR products were purified using the ChargeSwitch-Pro PCR Clean-up kit (Invitrogen, Carlsbad, CA, USA). The purified PCR products were sequenced and analyzed using an ABI PRISM Dye Terminator Cycle Sequencing FS Core kit (Applied Biosystems, USA) and an ABI 3100 DNA Sequencer (Applied Biosystems). The sequences obtained in this study have been registered in DDBJ/GenBank/EBI Data Bank under the accession numbers shown in Table 1.

\subsection{Sequence Alignments and Phylogenetic Analyses}

The nucleotide sequences were aligned using BioEdit software (version 6.0.8.0) [17]. Insertion-deletions (indels) were removed from all data set prior to phylogenetic analysis. Neighbor-joining (NJ) analyses were performed using MEGA 5 [18] by calculating genetic distance based on Kimura's two-parameter model [19]. One thousand bootstrap replications were performed for each tree to evaluate the reliability of the topology.

\section{Results and Discussion}

The intergenic spacer sequences of atpF-atpA in A. acutiloba varieties were 48 - 50 base pairs (bp) in length and contained two polymorphic sites (Table 2). The atpF-
atpA sequences were identical among all strains of $A$. acutiloba var. sugiyamae, A. acutiloba var. acutiloba from Yamagata, A. acutiloba var. iwatensis from Yamagata, A. stenoloba, A. stenoloba f. lanceolata, and one individual of $A$. shikokiana. There were intraspecific variations in atpF-atpA sequences in three strains of $A$. acutiloba var. acutiloba (TG, OT, and OC) and A. shikokiana. Two individuals of each of the TG (TG-1 and TG-2), OT (OT-1 and OT-2), and OC (OC-1 and OC-2) strains of A. acutiloba var. acutiloba contained indels of the T-nucleotide at $31 \mathrm{bp}$. There was also an indel of the T-nucleotide at $30 \mathrm{bp}$ between two individals of $\mathrm{A}$. shikokiana. Therefore, there were intraspecific variations in this region of the chloroplast genome both among and within strains of $A$. acutiloba var. acutiloba and A. shikokiana. It was previously reported that some varieties of A. acutiloba var. acutiloba (such as "Toki", "Yamato Toki" and "Ohbuka Toki") could be distinguished from other varieties (such as A. acutiloba var. sugiyamae and A. acutiloba var. iwatensis) by differences in the intergenic spacer sequences between the atpF and atpA genes [11]. In this study, however, the atpF-atpA region could not be used to authenticate $A$. acutiloba varieties and related species. The atpF-atpA sequence of Peucedanum japonicum (AB697546 in GenBank), which is a genus related to Angelica in the Umbelliferae, contained a 3-bp deletion between nucleotide positions 29 and 31, compared with the atpF-atpA sequences in Angelica species determined here. Therefore, this region may be useful to distinguish Angelica from related genera Peucedanum, but cannot be used to distinguish Angelica species from each other.

In three varieties of $A$. acutiloba, the sequences of the rpl16-rpl14 spacer region consisted of 463 bp that were identical to AB199891 in GenBank, which is the rpl16rpl14 region in A. acutiloba var. acutiloba. Although there were no variations in this region between $A$. acutiloba and A. stenoloba, A. stenoloba f. lanceolata contained a T-nucleotide insertion at nucleotide position 449 (Table 2). In A. shikokiana, there was a 31-bp insertion between nucleotides 365 and 396 in this region. A. shikokiana differs morphologically from A. acutiloba and $A$. stenoloba varieties, based on phenotypic traits. Therefore, the genetic background of A. shikokiana likely differs from that of the other species.

The trnK sequence was identical among A. acutiloba var. acutiloba from Gumma, Kyoto, Hokkaido, Miyagi, Toyama, and Chiba, and A. acutiloba var. iwatensis from Miyagi (Table 2). The trnK sequence in the Chinese $A$. acutiloba var. acutiloba had one base substitution at nucleotide position 2238. Thus, the Chinese A. acutiloba var. acutiloba could be distinguished from the others based on this sequence difference. In A. acutiloba var. 


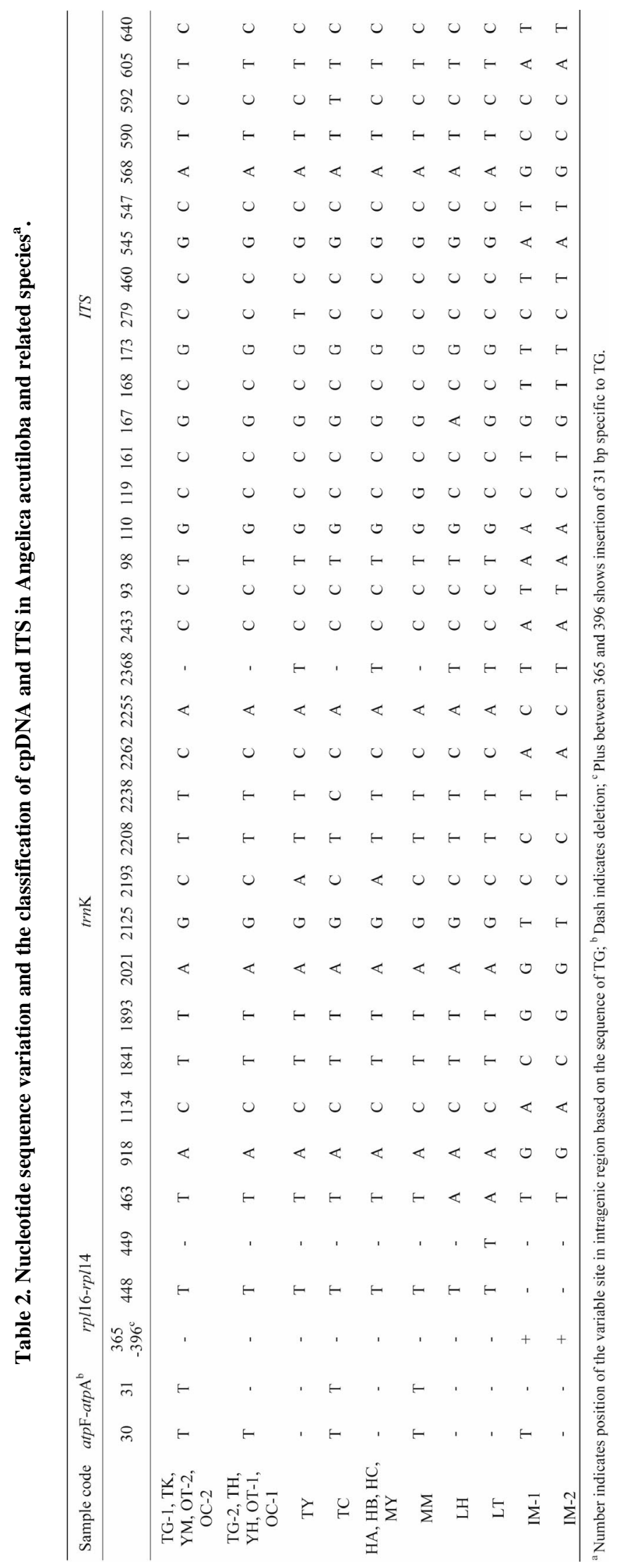




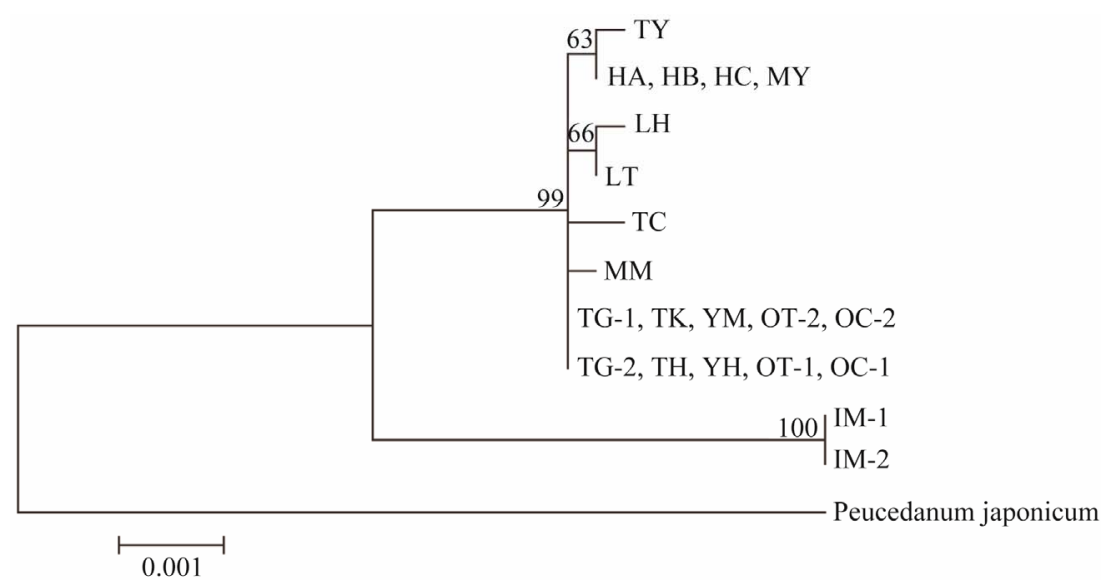

Figure 1. Neighbor-joining tree based on combined analysis of cpDNA (atpF-atpA, rpl16-rpl14, trnK) and ITS sequences. Tree was outgroup-rooted using sequence data from Peucedanum japonicum. Numbers beside internal branches indicate Bootstrap values $(>50 \%)$ calculated from 1000 replicates.

acutiloba from Yamagata, three strains of A. acutiloba var. sugiyamae, and A. acutiloba var. iwatensis from Yamagata, the trnK sequences had an $\mathrm{A}$ instead of a $\mathrm{C}$ at nucleotide position 2193, and often contained a T-nucleotide insertion at nucleotide position 2368. Two varieties of A. stenoloba and the "Hokkai Toki" variety also had the T-nucleotide insertion at nucleotide position 2368, and the latter had a T-to-A substitution at nucleotide position 463 . The trnK sequence in A. shikokiana had 10 base substitutions compared with that in A. acutiloba.

To obtain more information on genetic diversity among varieties and individuals of Angelica, especially among A. acutiloba varieties, we determined the sequences of the ITS region, including the 18S, ITS1, 5.8S, ITS2, and 26S regions. There were no variations in this region among individuals of $A$. acutiloba var. acutiloba (except for the strains from Yamagata and China), and all A. acutiloba var. sugiyamae. There was one unique substitution at position 279 in the $5.8 \mathrm{~S}$ region of A. acutiloba var. acutiloba from Yamagata, and a C-to-T substitution at position 592 in the ITS2 region of Chinese strain. Also, there was a one-nucleotide substitution at position 119 in the ITS1 region in A. acutiloba var. iwatensis from Miyagi and at position 167 in the ITS1 region in A. stenoloba. There were 13 nucleotide substitutions in the ITS sequence of A. shikokiana, compared with this region in the other varieties. The ITS region and the cpDNA sequence were identical between one strain of A. acutiloba var. iwatensis and all strains of A. acutiloba var. sugiyamae. These results imply that the 'Hokkai Toki' is derived from A. acutiloba var. iwatensis. In a previous study, A. acutiloba var. iwatensis from different geographical locations showed genetic polymorphisms in a RAPD analysis [9]. Because of that result and the differences in sequences of both the trnK and ITS regions, further analysis should be conducted using other A. acutiloba var. iwatensis varieties.

We constructed a neighbor-joining tree based on Kimura's two-parameter model [19] for the 10 haplotypes (Table 2) based on the combined analysis of cpDNA (atpF-atpA, rpl16-rpl14 and trnK) and ITS sequences (Figure 1). The monophyly of A. shikokiana and other species was supported by a bootstrap value of 100 . This result indicates that $A$. acutiloba is more closely related to two varieties of $A$. stenoloba than to A. shikokiana.

The traditional methods for authenticating crude drugs and adulterants are based on the morphological characters of the plants. In general, differences among morphological characters and compounds are subtle and ambiguous [20]. Based on sequence analyses of the trnK and ITS regions in A. acutiloba, we have proposed a method to authenticate the origin of Japanese Angelica Root; that is, whether it is derived from A. acutiloba var. acutiloba or A. acutiloba var. sugiyamae. The molecular authenticcation of these regions is a highly sensitive and stable method. The authentication results are reliable and are not affected by the physical form or physiological conditions of the plant samples. Therefore, the method based on the combined cpDNA and ITS sequences by the addition of conventional methods is useful for practical and accurate authentication of A. acutiloba var. acutiloba and A. acutiloba var. sugiyamae. This method will be useful in raw material production processes and for quality control of "Toki" produced for use in Kampo medicine in Japan.

\section{Acknowledgements}

We are grateful to Prof. K. Koike, Faculty of Pharmaceutical Sciences, Toho University, and Mr. H. Hayasaka, Graduate School of Pharmaceutical Science, Tohoku 
University, and Mr. M. Murakami, Toyama Prefectural Institute for Pharmaceutical Research, and Dr. T. Shibata, Research Center for Medicinal Plant Resources, National Institute of Biomedical Innovation, for cordially supplying A. acutiloba plants. We are also indebted to Assoc. Prof. K. Hotta, Faculty of Pharmaceutical Sciences, Health Sciences University of Hokkaido, for kindly providing A. acutiloba var. sugiyamae plants.

\section{REFERENCES}

[1] Hirokawa Publishing Co., "The Japanese Pharmacopoeia,” 16th Edition, Tokyo, 2011.

[2] E. Kaibara, “Chapter Galenicals Toki,” Yamato Honzo, Vol. 6, 1709.

[3] R. Terashima, "Chapter Herbs Toki," Wakansansazue, Vol. 93, 1713.

[4] H. Hikino, "Bibliographical Research and History of Productions of Toki or Tang Kuei 2,” Yakugaku Kenkyu, Vol. 29, 1957, pp. 1059-1078.

[5] Ministry of Health and Welfare, Pharmaceutical Affairs Bureau, "Yakuyou-Shokubutsu Saibai to HinshitsuHyoka Part 1,” Yakuji Nippo, Tokyo, 1992, pp. 41-50.

[6] T. Gotoh, T. Tanaka, T. Kawamura and Y. Noro, "Phytosociological Studies of the Communities on Angelica acutiloba and A. acutiloba var. iwatensis (1) Gifu Prefecture and Its Surroundings,” Natural Medicines, Vol. 49, 1995, pp. 255-260.

[7] K. Hatano, I. Nishioka and S. Iwasa, "Cytogenetical Studies of Umbelliferous Plants. I. 1) The Karyotype and Cross-Compatibility on the Original Plants of Japanese Toki,” Shoyakugaku Zasshi, Vol. 28, 1974, pp. 51-64.

[8] H. Mizukami, B. S. Hao and T. Tanaka, "Nucleotide Sequence of 5S-rDNA Intergenic Spacer Region in Angelica acutiloba," Natural Medicines, Vol. 51, 1997, pp. 376378.

[9] M. Kohjyouma, O. Iida, N. Yoshida, Y. Hatakeyama, M. Satake, S. Sekita and H. Kohda, "Random Amplified Polymorphic DNA Analysis of Angelica acutiloba and Its Varieties,” Natural Medicines, Vol. 52, 1998, pp. 130134.

[10] A. Watanabe, S. Araki, S. Kobari, H. Sudo, T. Tsuchida, T. Uno, N. Kosaka, K. Shimomura, M. Yamazaki and K. Saito, "In Vitro Propagation, Restriction Fragment Length Polymorphism, and Random Amplified Polymorphic DNA Analyses of Angelica Plants,” Plant Cell Reports, Vol. 18, No. 3-4, 1998, pp. 187-192. doi:10.1007/s002990050554

[11] K. Hosokawa, A. Hishida, I. Nakamura and T. Shibata,
"The Sequences of the Spacer Region between the atpF and atpA Genes in the Plastid Genome Allows Discrimination among Three Varieties of Medicinal Angelica," Planta Medica, Vol. 72, No. 6, 2006, pp. 570-571. doi:10.1055/s-2005-916257

[12] J. Murata, H. Oki, K. Kakutani and T. Hashimoto, "Identification of DNA polymorphisms in Angelica acutiloba," Plant Biotechnology, Vol. 25, No. 2, 2008, pp. 157-163. doi:10.5511/plantbiotechnology.25.157

[13] K. Fukuda, K. Murata, H. Matsuda and T. Tani, "History and the Present of Cultivation and Production of Angelica Root, Yamato-Toki, in Japan,” Yakushigaku Zasshi, Vol. 44, 2009, pp. 10-17.

[14] M. W. Lassner, P. Peterson and J. I. Yoder, "Simultaneous Amplification of Multiple DNA Fragments by Polymerase Chain Reaction in the Analysis of Transgenic Plants and Their Progeny,” Plant Molecular Biology Reporter, Vol. 7, No. 2, 1989, pp. 116-128. doi:10.1007/BF02669627

[15] S. Ohta, S. Osumi, T. Katsuki, I. Nakamura, T. Yamamoto and Y. Sato, "Genetic Characterization of Flowering Cherris (Prunus subgenus Cerasus) Using rpl16rpl14 Spacer Sequence of Chloroplast DNA,” Journal of the Japanese Society for Horticultural Science, Vol. 75, No. 1, 2006, pp. 72-78. doi:10.2503/jjshs.75.72

[16] S. Zhu, H. Fushimi, G. Han, T. Tsuchida, T. Uno, A. Takano and K. Komatsu, "Molecular Identification of 'Chuanxiong' by Nucleotide Sequence and Multiplex Single Base Extension Analysis of Chloroplast trnK Gene,” Biological and Pharmceutical Bulletin, Vol. 30, No. 3, 2007, pp. 527-531. doi:10.1248/bpb.30.527

[17] T. A. Hall, "BioEdit: A User-Friendly Biological Sequence Alignment Editor and Analysis Program for Windows 95/98/NT,” Nucleic Acids Symposium Series, Vol. 41, 1999, pp. 95-98.

[18] K. Tamura, D. Peterson, N. Peterson, G. Stecher, M. Nei and S. Kumar, "MEGA5: Molecular Evolutionary Genetics Analysis Using Maximum Likelihood, Evolutionary Distance, and Maximum Parsimony Methods,” Molecular Biology and Evolution, Vol. 28, 2011, pp. 2731-2739. doi:10.1093/molbev/msr121

[19] M. Kimura, “A Simple Method for Estimating Evolutionary Rates of Base Substitutions through Comparative Studies of Nucleotide Sequences," Journal of Molecular Evolution, Vol. 16, No. 2, 1980, pp. 111-120. doi:10.1007/BF01731581

[20] Z. Y. Yang, Z. Chao, K. K. Huo, H. Xie, Z. P. Tian and S. L. Pan, "ITS Sequence Analysis Used for Molecular Identification of the Bupleurum Species from Northwestern China,” Phytomedicine, Vol. 14, 2007, pp. 416-423. doi:10.1016/j.phymed.2007.04.009 